
\title{
COMPARISON OF ORTHODONTIC TREATMENT WITH DIFFERENT PREMOLAR
EXTRACTION MODALITIES IN TERMS OF SOFT TISSUE PROFILE
}

\begin{abstract}
Objectives: To evaluate the differences in changes in the soft tissue profile and dentoskeletal parameters between different premolar extraction and nonextraction treatment modalities.
\end{abstract}

Materials and Methods: Fifty patients with skeletal Class I malocclusion were divided into three groups. Group 1 consisted of 17 patients (mean age: $16.76 \pm 1.68$ years) treated with maxillary and mandibular first premolar extractions; Group 2 consisted of 16 patients (mean age: $15.81 \pm 1.19$ years) treated with maxillary and mandibular second premolar extractions, and Group 3 consisted of 17 patients (mean age:16.29 \pm 1.15 years) treated with non-extraction protocol. From the pre-treatment (T0) and post-treatment (T1) cephalometric radiographs, 13 measurements for dentoskeletal and 15 for soft tissue parameters were assessed. To determine changes due to treatment, and to compare differences among the groups, the Wilcoxon Signed-Rank and Kruskal-Wallis tests were performed, respectively.

Results: Mx1-SN, Mx1-FH, Mx1-NA, IMPA and Md1-NB values decreased significantly in Groups 1 and 2 compared to Group 3 ( $\mathrm{p}<0.001)$. According to the vertical reference line (VRL-li) and E-plane (E-LL), the lower lip showed a statistically significant change (retraction) in Group 1 and 2, compared to the non-extraction group $(\mathrm{p}<0.05)$. The mean change value for the upper and lower lip thicknesses in Groups 1 and 2 was greater than in Group $3(p<0.05)$. Groups 1 and 2 did not show a significant difference in any dentoskeletal or soft tissue measurements.

Conclusions: Soft tissue profile change following extraction treatment was similar regardless of the extracted teeth. However, extraction treatment modalities resulted in significant profile changes especially in the lower lip compared to the non-extraction control group.
D Ezgi ATİK ${ }^{1}$

(D)*Hande GÖRÜCÜ COŞKUNER ${ }^{1}$

D Tülin TANER ${ }^{1}$

ORCID IDs of the authors: E.A. 0000-0002-5912-4505

H.G.C. 0000-0001-7426-6731

T.T. 0000-0003-1358-0633

1 Department of Orthodontics, Faculty of Dentistry, Hacettepe University, Ankara, Turkey

Keywords: Soft tissue, tooth extraction, vertical dimension.

How to Cite: Atik E, Görücü Coşkuner H, Taner T. Comparison of Orthodontic Treatment with Different Premolar Extraction Modalities in Terms of Soft Tissue Profile. Cumhuriyet Dent J 2019;22:4

*Corresponding Author:

Department of Orthodontics, Faculty of Dentistry, Hacettepe University, Sihhiye, 6100, Ankara/Turkey

Phone: +903123052290 Fax: +903123091138 Email: hande.gorucu@hotmail.com 


\section{INTRODUCTION}

Orthodontic treatments with extraction are treatment approaches that significantly affect the patient's profile and aesthetics. ${ }^{1}$ One of the most controversial issues in the field of orthodontics is whether to use treatment alternatives with extraction or non-extraction. The choice between extraction and non-extraction treatment modalities has a considerable impact on numerous parameters, such as treatment stability, vertical dimension, arch width, soft tissues and facial convexity. ${ }^{2-5}$ One of the most important reasons for this discussion is the possible effect of these two different treatment methods on the soft tissue. In many studies ${ }^{6-8}$, it has been emphasized that orthodontic treatments, including premolar extractions, may cause lip retraction, and a significant change in the facial profile compared to non-extraction treatment approaches. However, contrary to this view, other authors have stated that the withdrawal treatments will not have a negative effect on the soft tissue profile. ${ }^{9-12}$

Another effect area of orthodontic treatments on the face is the change in vertical height. According to the "wedge-type effect," extracting permanent posterior teeth may reduce the vertical dimension of the face by counterclockwise rotation of the mandible, through the forward movement of the posterior teeth. ${ }^{13}$ It has been hypothesized that second premolar extractions allow the molar teeth to move more forward when compared to first premolar extractions, thus resulting in a greater decrease of the facial vertical dimension. ${ }^{14-16}$

In the literature, several studies have compared various extraction treatment protocols to nonextraction treatment. ${ }^{2,17-20}$ However, there is a limited number of studies comparing the extraction of the four first premolar teeth with the four second premolar teeth regarding the amount of soft-tissue and vertical facial height changes performed during treatment. ${ }^{21-22}$ Thus, in this retrospective observational study, we aimed to determine whether the removal of four first premolar teeth resulted in any differences in the soft tissue profile and vertical facial height change compared to the removal of four second premolar teeth using a non-extraction control group. The null hypothesis of the present study was that there is no statistically significant difference in soft tissue and vertical facial height changes when comparing the extraction of four first premolars to four second premolars in patients treated with different extraction modalities.

\section{MATERIALS AND METHODS}

The treatment records of 50 patients with skeletal Class I malocclusion were selected from the archive of the Department of Orthodontics, Faculty of Dentistry, Hacettepe University. The Institutional Ethics Committee of Hacettepe University (GO 19/21) approved the study design. The inclusion criteria for this retrospective study were as follows: 1) Lateral cephalometric images of patients who had been treated with four first or second premolar extraction with moderate anchorage mechanics or without extraction; 2) Use of a transpalatal arch and a Nance appliance in the maxilla and a lingual arch in the mandible for anchorage in patients who underwent extraction treatment; 3) Images of patients with high-quality pre- and post-treatment lateral cephalometric films exhibiting relaxed lips and teeth in occlusion, taken using the same cephalostat; 4) Images of patients with skeletal Class I malocclusion and moderate maxillary and mandibular crowding at the beginning of treatment; 5) Images of patients over 14 years of age; and 6) Images of patients with no craniofacial anomalies, and all teeth, excluding third molars, were present.

The dental files of 115 patients, who had been treated with extraction of either the four first or four second premolars, were reviewed. Of 115 records, 33 patients treated with the extraction protocol were included for this study. The final sample size per group was determined by the maximum number of cases eligible according to the strict inclusion criteria.

Group 1 consisted of 17 patients (12 female, 5 male) with a mean age of 16.76 years, who had been treated with four first premolar extractions. Group 2 consisted of 16 patients (10 female, 6 male) with a mean age of 15.81 years, who had been treated with four second premolar extractions. Group 3 consisted of 17 patients (13 female, 4 
male) with a mean age of 16.29 years, with moderate maxillary and mandibular anterior crowding, and had been treated with the nonextraction treatment to serve as a control group. We aimed to make the clinical and demographic characteristics of the control group similar to the extraction groups. All patients were treated with pre-adjusted Roth prescription 0.018-inch appliances in both arches. In the extraction groups, premolar teeth were extracted to relieve crowding, the reduction of incisor protrusions, and/or the reduction of lip protrusion. In these groups, moderate anchorage mechanics, including the Nance appliance and a transpalatal arch for the maxilla; a lingual arch for the mandible was preferred. Crowding was initially alleviated by retraction of the canines, and the remaining space was closed by reciprocal traction of the posterior segment.

In the extraction groups, space closure was performed with sliding mechanics combined with a stainless steel (SS) archwire of $0.016 \times 0.016$ inch. The canine teeth in Group 1 and the canine and first premolar teeth in Group 2 were retracted through an archwire, using coil springs with sliding mechanics. After the canine teeth were retracted, the anchorage devices were removed, and $0.016 \times 0.022$-inch SS archwires with T-loops were used to close the remaining spaces. In the non-extraction group, crowding was eliminated by the expansion of the arches and/or proclination of the incisors. In the cases needing expansion, a Quad-Helix was used as an expansion device.

Lateral cephalometric radiographs were taken with a cephalostat (Promax; Planmeca, Helsinki, Finland) before (T0) and after (T1) treatment. Each subject's pre-treatment and post-treatment lateral cephalograms were traced using the Quick Ceph Studio software (Quick Ceph Systems, San Diego, Calif) by one examiner (E. A.) and were reviewed twice by another investigator (H. G-C.) for accurate landmark identification. Twenty-five subjects were randomly selected and retraced 2 weeks later by the same investigator to evaluate intra-examiner reliability.

For the analysis of soft tissue measurements, two reference lines were created: a horizontal reference line through Sella seven degrees inferior to the Sella-Nasion line, and a vertical reference line (VRL) through Sella perpendicular to SN$7^{\circ} .{ }^{23}$ Twelve linear and 3 angular measurements were constructed for soft tissue measurements (Figure 1).

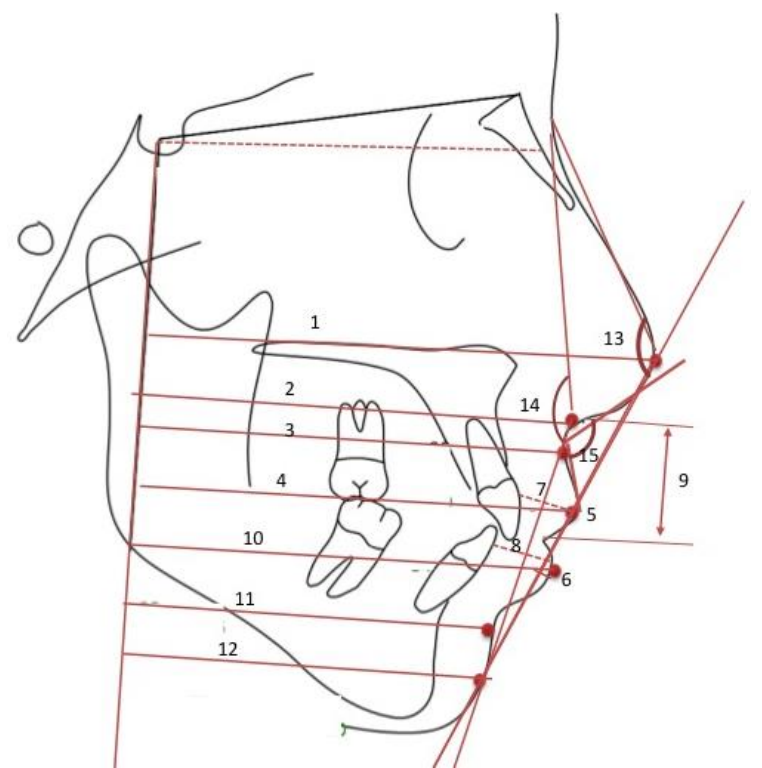

Figure 1. a. Linear measurements of the soft tissue: 1- VRL-prn; 2- VRLsn; 3- VRL-A; 4-VRL-ls; 5-E-UL; 6-E-LL; 7-U1-ls; 8-L1-ls; 9-upper lip length; 10-VRL-li; 11-VRL-B'; 12-VRL-pog'. b. Angular measurements of the soft tissue: 13-na-prn-pog ${ }^{\circ}$; 14-na-sn-pog ${ }^{\circ} ; 15$-nasolabial angle.

For skeletal and dental measurements, 9 angular and 4 linear cephalometric variables were recorded (Figure 2).

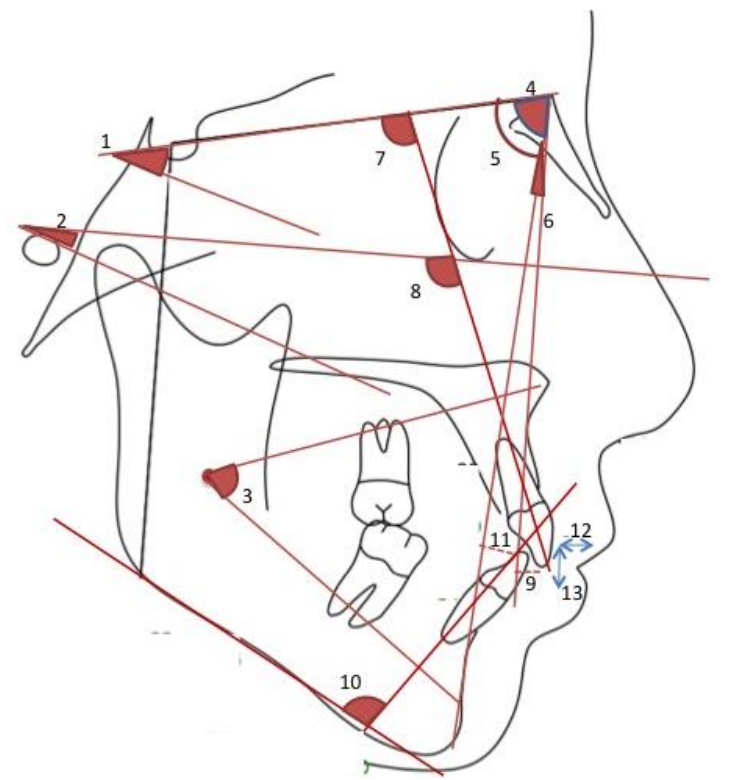

Figure 2. a. Vertical measurements of the hard tissue: $1-\mathrm{GoGnSN}^{\circ} ; 2-$ FMA $^{\circ} ; 3-\mathrm{ANS}-\mathrm{Xi}-\mathrm{Pm}^{\circ}$. b. Other skeletal and dental measurements of the hard tissue: $4-\mathrm{SNA}^{\circ} ; 5-\mathrm{SNB}^{\circ} ; 6-\mathrm{ANB}^{\circ} ; 7-\mathrm{Mx}^{\circ}-\mathrm{SN}^{\circ} ; 8-\mathrm{Mx}^{\circ}-\mathrm{FH}^{\circ} ; 9-\mathrm{Mx} 1-\mathrm{NA}$ $(\mathrm{mm}) ; 10-\mathrm{IMPA}^{\circ} ; 11-\mathrm{Md} 1-\mathrm{NB}(\mathrm{mm}) ; 12$-Overjet (mm); 13-Overbite $(\mathrm{mm})$.

\section{Statistical Analysis:}

Statistical analyses were performed using SPSS software for Windows (version 21; IBM, Chicago, IL, USA). The descriptive data were presented as 
frequency (\%) or mean \pm standard deviation. Group comparability regarding gender and cervical vertebral maturation stage (CVMS) was evaluated with chi-square analysis. Since all data were non-normally distributed, the Kruskal-Wallis nonparametric test was used to compare of the groups regarding age, treatment duration, maxillary crowding amount, mandibular crowding amount, and initial cephalometric measurements. Also, for the comparison of difference related to hard and soft tissue measurements between T0 and T1, the Kruskal-Wallis test was used. The Bonferroni Dunn Test was used to reveal the group that created the difference.
The Wilcoxon Signed Rank test was used to determine the significance of cephalometric measurement changes from $\mathrm{T} 0$ to $\mathrm{T} 1$ within the groups. Intra-examiner reliability was measured with Intraclass Correlation Coefficient (ICC) analysis. The significance level was set at $\mathrm{p}<0.05$ for all the tests.

\section{RESULTS}

The ICC values were between 0.892 and 0.996 , which were within acceptable limits. The pretreatment demographic characteristics are shown in Table 1.

Table 1. Demographic characteristics of the sample in different groups.

\begin{tabular}{|c|c|c|c|c|}
\hline Variables & $\begin{array}{c}\text { Group } 1(\mathrm{~N}=17) \\
\text { Mean } \pm \text { SD or } \\
\text { Number (frequency \%) }\end{array}$ & $\begin{array}{c}\text { Group } 2(\mathrm{~N}=16) \\
\text { Mean } \pm \text { SD or Number } \\
(\text { frequency } \%)\end{array}$ & $\begin{array}{c}\text { Group } 3(\mathrm{~N}=17) \\
\text { Mean } \pm \text { SD or Number } \\
(\text { frequency } \%)\end{array}$ & p-value \\
\hline Age (years) & $16.76 \pm 1.68$ & $15.81 \pm 1.19$ & $16.29 \pm 1.15$ & $0.118^{\mathrm{a}}$ \\
\hline \multicolumn{5}{|l|}{ Gender } \\
\hline $\begin{array}{l}\text { Female } \\
\text { Male }\end{array}$ & $\begin{array}{c}12(70.6 \%) \\
5(29.4 \%)\end{array}$ & $\begin{array}{c}10(62.5 \%) \\
6(37.5 \%)\end{array}$ & $\begin{array}{c}13(76.5 \%) \\
4(23.5 \%)\end{array}$ & $0.680^{\mathrm{b}}$ \\
\hline CVMS period & & & & \\
\hline $\begin{array}{l}\text { CVMS } 4 \\
\text { CVMS } 5 \\
\text { CVMS } 6\end{array}$ & $\begin{array}{l}3(17.6 \%) \\
6(35.3 \%) \\
8(47.1 \%)\end{array}$ & $\begin{array}{l}7(43.8 \%) \\
7(43.8 \%) \\
2(12.5 \%)\end{array}$ & $\begin{array}{l}3(17.6 \%) \\
5(29.4 \%) \\
9(52.9 \%)\end{array}$ & $0.115^{\mathrm{b}}$ \\
\hline $\begin{array}{l}\text { Treatment } \\
\text { duration (years) }\end{array}$ & $2.18 \pm 0.64$ & $2.03 \pm 0.50$ & $1.16 \pm 0.43$ & $\begin{array}{c}<0.001^{\mathrm{a}} \\
(1-3) \\
(2-3)\end{array}$ \\
\hline $\begin{array}{l}\text { Maxillary } \\
\text { crowding (mm) }\end{array}$ & $5.65 \pm 1.22$ & $5.44 \pm 1.39$ & $5.53 \pm 0.51$ & 0.891 \\
\hline $\begin{array}{l}\text { Mandibular } \\
\text { crowding }(\mathbf{m m})\end{array}$ & $5.18 \pm 1.27$ & $4.94 \pm 1.03$ & $4.85 \pm 0.95$ & 0.789 \\
\hline
\end{tabular}

a: Kruskal-Wallis test, b:chi-square test

$\mathrm{p}<0.05$ is statistically significant.

(1-3): $\mathrm{p}$ value for difference between Group 1 and 3

(2-3): $\mathrm{p}$ value for difference between Group 2 and 3 .

There were no significant differences in any demographic characteristics, cephalometric or model measurements among the 3 groups except for treatment duration, which was significantly

lower in Group 3 compared to Groups 1 and 2 $(\mathrm{p}<0.001)$. Skeletal, dental, and soft tissue measurement differences at pre-treatment among the 3 groups are shown in Table 2. 
Atik E, et al.

Table 2. Pre-treatment (T0) differences between the groups with respect to hard and soft tissue cephalometric measurements.

\begin{tabular}{|c|c|c|c|c|}
\hline Variables & $\begin{array}{c}\text { Group } 1(\mathbf{N}=17) \\
\text { Mean } \pm \text { SD }\end{array}$ & $\begin{array}{c}\text { Group } 2(\mathrm{~N}=16) \\
\text { Mean } \pm \text { SD }\end{array}$ & $\begin{array}{c}\text { Group } 3(\mathbf{N}=17) \\
\text { Mean } \pm \text { SD }\end{array}$ & p-value \\
\hline $\mathrm{ANB}^{\circ}$ & $3.28 \pm 1.01$ & $2.59 \pm 0.99$ & $3.29 \pm 0.83$ & 0.085 \\
\hline $\mathrm{Mx} 1-\mathrm{SN}^{\circ}$ & $106.39 \pm 6.35$ & $106.87 \pm 6.22$ & $105.55 \pm 6.48$ & 0.898 \\
\hline $\mathrm{Mx} 1-\mathrm{FH}^{\circ}$ & $116.46 \pm 5.69$ & $116.11 \pm 5.03$ & $115.49 \pm 6.46$ & 0.962 \\
\hline Mx1-NA (mm) & $6.94 \pm 2.36$ & $6.44 \pm 1.60$ & $5.37 \pm 2.24$ & 0.228 \\
\hline IMPA $^{\circ}$ & $103.98 \pm 6.36$ & $96.24 \pm 7.12$ & $99.91 \pm 8.39$ & $\begin{array}{c}0.017^{*} \\
(1-2=0.013)\end{array}$ \\
\hline Md1-NB (mm) & $8.14 \pm 1.61$ & $7.08 \pm 2.51$ & $6.74 \pm 2.05$ & 0.083 \\
\hline Overjet (mm) & $3.73 \pm 1.61$ & $3.44 \pm 1.24$ & $3.98 \pm 0.93$ & 0.309 \\
\hline Overbite (mm) & $1.00 \pm 1.53$ & $1.18 \pm 1.09$ & $2.20 \pm 1.04$ & $\begin{array}{c}0.012 * \\
(1-3=0.017)\end{array}$ \\
\hline $\mathrm{GoGnSN}^{\circ}$ & $33.56 \pm 5.10$ & $37.36 \pm 4.89$ & $32.45 \pm 5.06$ & $\begin{array}{c}0.017 * \\
(2-3=0.017) \\
0.001 *\end{array}$ \\
\hline $\mathrm{FMA}^{\circ}$ & $23.77 \pm 4.93$ & $28.10 \pm 3.32$ & $22.49 \pm 4.82$ & $\begin{array}{l}(1-2=0.006) \\
(2-3=0.001)\end{array}$ \\
\hline ANS-Xi-Pm ${ }^{\circ}$ & $48.68 \pm 4.92$ & $49.02 \pm 3.51$ & $45.84 \pm 3.19$ & 0.066 \\
\hline VRL-prn (mm) & $97.22 \pm 6.88$ & $94.75 \pm 3.55$ & $94.28 \pm 4.99$ & 0.386 \\
\hline VRL-sn (mm) & $82.43 \pm 7.70$ & $80.09 \pm 5.11$ & $80.38 \pm 4.00$ & 0.461 \\
\hline VRL-A' (mm) & $80.64 \pm 7.34$ & $78.28 \pm 4.91$ & $77.93 \pm 4.03$ & 0.444 \\
\hline VRL-ls (mm) & $82.65 \pm 7.95$ & $80.74 \pm 5.68$ & $79.55 \pm 5.25$ & 0.518 \\
\hline E-UL (mm) & $-3.26 \pm 1.94$ & $-3.36 \pm 2.35$ & $-3.93 \pm 2.44$ & 0.542 \\
\hline E-LL (mm) & $-1.28 \pm 2.19$ & $-0.87 \pm 2.02$ & $-2.20 \pm 1.84$ & 0.142 \\
\hline U1-ls (mm) & $11.32 \pm 1.71$ & $11.80 \pm 2.20$ & $12.15 \pm 2.24$ & 0.631 \\
\hline L1-li (mm) & $12.27 \pm 2.30$ & $13.31 \pm 1.39$ & $12.68 \pm 2.37$ & 0.324 \\
\hline upper lip length (mm) & $21.52 \pm 2.29$ & $22.24 \pm 3.61$ & $19.75 \pm 1.99$ & $\begin{array}{c}0.032 * \\
(2-3=0.052)\end{array}$ \\
\hline VRL-li & $79.69 \pm 8.88$ & $78.14 \pm 5.46$ & $77.04 \pm 5.86$ & 0.693 \\
\hline VRL-B' (mm) & $71.49 \pm 9.18$ & $69.44 \pm 5.76$ & $68.71 \pm 6.28$ & 0.726 \\
\hline VRL-pog' (mm) & $70.44 \pm 10.37$ & $68.64 \pm 6.07$ & $68.56 \pm 7.34$ & 0.929 \\
\hline na-prn-pog(mm) & $127.35 \pm 3.44$ & $129.56 \pm 2.87$ & $126.52 \pm 4.10$ & 0.068 \\
\hline na-sn-pog $(\mathrm{mm})$ & $157.78 \pm 5.14$ & $159.54 \pm 5.70$ & $156.18 \pm 4.82$ & 0.244 \\
\hline nasolabial angle & $103.87 \pm 13.39$ & $105.56 \pm 11.76$ & $105.81 \pm 9.02$ & 0.922 \\
\hline
\end{tabular}

Kruskal-Wallis test was used for intergroup comparisons, and Bonferroni Dunn test for post-hoc analysis.

(1-3): $\mathrm{p}$ value for difference between Group 1 and 3, (2-3): $\mathrm{p}$ value for difference between Group 2 and 3.

Significant differences in skeletal and dental measurements were found in $\mathrm{IMPA}^{\circ}$, overbite $(\mathrm{mm}), \mathrm{GoGnSN}^{\circ}$, and $\mathrm{FMA}^{\circ}$ values $(\mathrm{p}<0.05)$. Pre-treatment soft tissue measurements did not differ among the groups, except for upper lip length, which was higher in Group 2 compared to Group 3 ( $\mathrm{p}=0.052$ ).

Table 3 shows the mean values for pretreatment and post-treatment skeletal and dental measurements and the significance of changes during treatment in each group. $\mathrm{Mx} 1-\mathrm{SN}^{\circ}, \mathrm{Mx} 1-$ $\mathrm{FH}^{\circ}, \mathrm{Mx} 1-\mathrm{NA}(\mathrm{mm}), \mathrm{IMPA}^{\circ}, \mathrm{Md1-NB}(\mathrm{mm})$ values decreased significantly in Groups 1 and 2, while these values significantly increased in Group 3 ( $\mathrm{p}<0.05)$. Overbite was significantly increased in Groups 1 and 2, but was significantly decreased in Group $3(\mathrm{p}<0.05)$. Vertical skeletal variables $\left(\mathrm{GoGnSN}^{\circ}, \mathrm{FMA}^{\circ}\right.$, and $\left.\mathrm{ANS}-\mathrm{Xi}-\mathrm{Pm}^{\circ}\right)$ did not show significant changes from pretreatment to the post-treatment period in all groups. The decreases in maxillary and mandibular incisor inclinations in Groups 1 and 2 were significantly different from the increase of these values in Group 3 ( $p<0.05)$. 
Table 3. Pre-treatment (T0) and post-treatment (T1) skeletal and dental measurements, changes during treatment in each group, and intergroup comparisons.

\begin{tabular}{|c|c|c|c|c|c|c|c|c|}
\hline Variables & T0/T1 & $\begin{array}{c}\text { Group 1 } \\
(\mathbf{N}=17) \\
\text { Mean } \pm \text { SD } \\
\end{array}$ & $\begin{array}{c}\text { p-value } \\
\text { (Group } 1 \\
\text { T1-T0) } \\
\end{array}$ & $\begin{array}{c}\text { Group 2 } \\
(\mathbf{N}=\mathbf{1 6}) \\
\operatorname{Mean} \pm \text { SD } \\
\end{array}$ & $\begin{array}{c}\text { p-value } \\
\text { (Group } 2 \\
\text { T1-T0) } \\
\end{array}$ & $\begin{array}{c}\text { Group 3 } \\
(\mathbf{N}=17) \\
\operatorname{Mean} \pm \text { SD } \\
\end{array}$ & $\begin{array}{c}\text { p-value } \\
\text { (Group } 3 \\
\text { T1-T0) } \\
\end{array}$ & $\begin{array}{c}\begin{array}{c}\text { Intergroup } \\
\text { comparison } \\
\text { p-value }\end{array} \\
\end{array}$ \\
\hline \multirow{3}{*}{$\mathrm{Mx} 1-\mathrm{SN}^{\circ}$} & T0 & $106.39 \pm 6.35$ & \multirow{3}{*}{$<0.001 *$} & $106.87 \pm 6.22$ & \multirow{3}{*}{$0.003^{*}$} & $105.55 \pm 6.48$ & \multirow{3}{*}{$<0.001^{*}$} & $<0.001 *$ \\
\hline & $\mathrm{T} 1$ & $98.60 \pm 7.85$ & & $100.07 \pm 5.85$ & & $110.92 \pm 4.88$ & & $(1-3<0.001)$ \\
\hline & T1-T0 & $-7.79 \pm 3.92$ & & $-6.80 \pm 6.29$ & & $5.37 \pm 4.71$ & & $(2-3<0.001)$ \\
\hline \multirow{3}{*}{$\mathrm{Mx} 1-\mathrm{FH}^{\circ}$} & T0 & $116.46 \pm 5.69$ & \multirow{3}{*}{$<0.001^{*}$} & $116.11 \pm 5.03$ & \multirow{3}{*}{$0.007 *$} & $115.49 \pm 6.46$ & \multirow{3}{*}{$<0.001^{*}$} & $<0.001^{*}$ \\
\hline & $\mathrm{T} 1$ & $109.28 \pm 7.41$ & & $110.16 \pm 5.16$ & & $120.69 \pm 3.84$ & & $(1-3<0.001)$ \\
\hline & T1-T0 & $-7.18 \pm 4.30$ & & $-5.95 \pm 6.41$ & & $5.20 \pm 4.40$ & & $(2-3<0.001)$ \\
\hline \multirow{3}{*}{$\begin{array}{l}\text { Mx1-NA } \\
(\mathrm{mm})\end{array}$} & T0 & $6.94 \pm 2.36$ & \multirow{3}{*}{$<0.001 *$} & $6.44 \pm 1.60$ & \multirow{3}{*}{$0.004 *$} & $5.37 \pm 2.24$ & \multirow{3}{*}{$0.001^{*}$} & $<0.001 *$ \\
\hline & $\mathrm{T} 1$ & $2.91 \pm 2.49$ & & $4.30 \pm 2.03$ & & $6.61 \pm 2.05$ & & $(1-3<0.001)$ \\
\hline & T1-T0 & $-4.04 \pm 2.71$ & & $-2.14 \pm 2.13$ & & $1.24 \pm 0.80$ & & $(2-3=0.001)$ \\
\hline \multirow{3}{*}{ IMPA $^{\circ}$} & T0 & $103.98 \pm 6.36$ & \multirow{3}{*}{$<0.001^{*}$} & $96.24 \pm 7.12$ & \multirow{3}{*}{$0.011 *$} & $99.91 \pm 8.39$ & \multirow{3}{*}{0.004} & $<0.001 *$ \\
\hline & $\mathrm{T} 1$ & $94.97 \pm 7.31$ & & $90.46 \pm 7.56$ & & $103.94 \pm 8.42$ & & $(1-3<0.001)$ \\
\hline & T1-T0 & $-9.01 \pm 4.68$ & & $-5.78 \pm 7.49$ & & $4.03 \pm 4.91$ & & $(2-3=0.002)$ \\
\hline \multirow{3}{*}{$\begin{array}{l}\text { Md1-NB } \\
(\mathrm{mm})\end{array}$} & T0 & $8.14 \pm 1.61$ & \multirow{3}{*}{$<0.001 *$} & $7.08 \pm 2.51$ & \multirow{3}{*}{$0.012 *$} & $6.74 \pm 2.05$ & \multirow{3}{*}{$<0.001^{*}$} & $<0.001 *$ \\
\hline & $\mathrm{T} 1$ & $5.10 \pm 1.73$ & & $5.71 \pm 2.29$ & & $8.42 \pm 1.86$ & & $(1-3<0.001)$ \\
\hline & T1-T0 & $-3.04 \pm 1.34$ & & $-1.38 \pm 1.74$ & & $1.69 \pm 1.15$ & & $(2-3=0.001)$ \\
\hline \multirow{3}{*}{$\begin{array}{l}\text { Overjet } \\
(\mathrm{mm})\end{array}$} & T0 & $3.73 \pm 1.61$ & \multirow{3}{*}{0.469} & $3.44 \pm 1.24$ & \multirow{3}{*}{0.395} & $3.98 \pm 0.93$ & \multirow{3}{*}{$0.028^{*}$} & \multirow{3}{*}{0.751} \\
\hline & $\mathrm{T} 1$ & $3.47 \pm 0.69$ & & $3.13 \pm 0.94$ & & $3.36 \pm 1.01$ & & \\
\hline & T1-T0 & $-0.27 \pm 1.40$ & & $-0.32 \pm 1.44$ & & $-0.62 \pm 1.02$ & & \\
\hline \multirow{3}{*}{$\begin{array}{l}\text { Overbite } \\
(\mathrm{mm})\end{array}$} & T0 & $1.00 \pm 1.53$ & \multirow{3}{*}{$0.012 *$} & $1.18 \pm 1.09$ & \multirow{3}{*}{$0.013 *$} & $2.20 \pm 1.04$ & \multirow{3}{*}{$0.004^{*}$} & $<0.001 *$ \\
\hline & $\mathrm{T} 1$ & $2.00 \pm 0.98$ & & $1.90 \pm 0.80$ & & $1.29 \pm 0.91$ & & $(1-3<0.001)$ \\
\hline & T1-T0 & $0.99 \pm 1.43$ & & $0.72 \pm 0.92$ & & $-0.91 \pm 0.97$ & & $(2-3=0.001)$ \\
\hline & T0 & $33.56 \pm 5.10$ & & $37.36 \pm 4.89$ & & $32.45 \pm 5.06$ & & \\
\hline $\mathrm{GoGnSN}^{\circ}$ & $\mathrm{T} 1$ & $33.39 \pm 5.21$ & 0.619 & $37.91 \pm 4.19$ & 0.315 & $32.92 \pm 5.38$ & 0.236 & 0.433 \\
\hline & T1-T0 & $-0.17 \pm 1.96$ & & $0.55 \pm 2.49$ & & $0.47 \pm 1.46$ & & \\
\hline & T0 & $23.77 \pm 4.93$ & & $28.10 \pm 3.32$ & & $22.49 \pm 4.82$ & & \\
\hline FMA $^{\circ}$ & $\mathrm{T} 1$ & $23.07 \pm 5.08$ & 0.266 & $27.80 \pm 3.02$ & 0.517 & $23.18 \pm 5.16$ & 0.084 & 0.110 \\
\hline & T1-T0 & $-0.70 \pm 2.23$ & & $-0.30 \pm 1.90$ & & $0.68 \pm 1.62$ & & \\
\hline & T0 & $48.68 \pm 4.92$ & & $49.02 \pm 3.51$ & & $45.84 \pm 3.19$ & & \\
\hline $\begin{array}{l}\mathrm{Pm}^{\circ} \\
\end{array}$ & $\mathrm{T} 1$ & $48.03 \pm 5.04$ & 0.443 & $49.36 \pm 3.57$ & 0.315 & $45.71 \pm 3.87$ & 0.766 & 0.456 \\
\hline & T1-T0 & $-0.65 \pm 2.45$ & & $0.34 \pm 1.35$ & & $-0.14 \pm 1.86$ & & \\
\hline
\end{tabular}

Wilcoxon signed rank test was used for intragroup comparisons, Kruskal-Wallis test was used for intergroup comparisons, and Bonferroni Dunn test for post-hoc analysis (1-2): $\mathrm{p}$ value for difference between group 1 and 2, (1-3): $\mathrm{p}$ value for difference between group 1 and $3,(2-3)$ : $\mathrm{p}$ value for difference between group 2 and 3 . $\mathrm{p}<0.05$ is statistically significant.

The pre-treatment (T0) and post-treatment (T1) soft tissue measurements, changes during treatment in each group, and intergroup comparisons are shown in Table 4. In relation to the E-plane, the upper lip was significantly retracted $(\mathrm{p}=0.027)$ in Group 1, while the lower lip was significantly retracted in both Group 1 $(\mathrm{p}=0.003)$ and Group $2(\mathrm{p}=0.008)$. Upper lip thicknesses (U1-ls) showed a statistically significant increase from $\mathrm{T} 0$ to $\mathrm{T} 1$ in both Groups
1 and $2(p \leq 0.001)$. Lower lip thickness (L1-li) significantly increased $(\mathrm{p}=0.032)$ in Group 1, whereas it was decreased in Group $3(\mathrm{p}=0.029)$. Soft tissue measurements related to the lower lip and pogonion (VRL-li, VRL-B', VRL-pog') significantly moved anteriorly in Group 3 $(p<0.05)$. VRL-li distance significantly decreased $(\mathrm{p}=0.01)$ in Group 1. The nasolabial angle significantly decreased ( $\mathrm{p}=0.039$ ) in Group 3. 
Table 4. Pre-treatment (T0) and post-treatment (T1) soft tissue measurement changes during treatment in each group, and intergroup comparisons.

\begin{tabular}{|c|c|c|c|c|c|c|c|c|}
\hline Variables & T0/T1 & $\begin{array}{c}\text { Group 1 } \\
(\mathrm{N}=17) \\
\operatorname{Mean} \pm \mathrm{SD}\end{array}$ & $\begin{array}{c}\text { p-value } \\
\text { (Group } 1 \\
\text { T1-T0) }\end{array}$ & $\begin{array}{c}\text { Group } 2 \\
(\mathrm{~N}=16) \\
\operatorname{Mean} \pm \mathrm{SD}\end{array}$ & $\begin{array}{c}\text { p-value } \\
\text { (Group } 2 \\
\text { T1-T0) }\end{array}$ & $\begin{array}{c}\text { Group } 3 \\
(\mathrm{~N}=17) \\
\operatorname{Mean} \pm \mathbf{S D}\end{array}$ & $\begin{array}{c}\text { p-value } \\
\text { (Group } 3 \\
\text { T1-T0) }\end{array}$ & $\begin{array}{c}\text { Intergroup } \\
\text { comparison } \\
\text { p-value }\end{array}$ \\
\hline \multirow{3}{*}{$\begin{array}{l}\text { VRL-prn } \\
(\mathrm{mm})\end{array}$} & T0 & $97.22 \pm 6.88$ & \multirow{3}{*}{0.501} & $94.75 \pm 3.55$ & & $94.28 \pm 4.99$ & \multirow{3}{*}{0.286} & \multirow{3}{*}{0.128} \\
\hline & $\mathrm{T} 1$ & $96.95 \pm 7.13$ & & $95.50 \pm 4.93$ & 0.162 & $94.62 \pm 5.31$ & & \\
\hline & T1-T0 & $-0.27 \pm 1.29$ & & $0.75 \pm 1.93$ & & $0.34 \pm 1.34$ & & \\
\hline \multirow{3}{*}{$\begin{array}{l}\text { VRL-sn } \\
(\mathrm{mm})\end{array}$} & T0 & $82.43 \pm 7.70$ & \multirow{3}{*}{0.868} & $80.09 \pm 5.11$ & \multirow{3}{*}{0.477} & $80.38 \pm 4.00$ & \multirow{3}{*}{0.266} & \multirow{3}{*}{0.859} \\
\hline & $\mathrm{T} 1$ & $82.59 \pm 7.10$ & & $80.51 \pm 5.66$ & & $80.65 \pm 4.32$ & & \\
\hline & T1-T0 & $017 \pm 2.35$ & & $0.42 \pm 3.00$ & & $0.27 \pm 1.15$ & & \\
\hline \multirow{3}{*}{$\begin{array}{l}\text { VRL-A' } \\
(\mathrm{mm})\end{array}$} & T0 & $80.64 \pm 7.34$ & \multirow{3}{*}{0.213} & $78.28 \pm 4.91$ & \multirow{3}{*}{0.570} & $77.93 \pm 4.03$ & \multirow{3}{*}{0.133} & \multirow{3}{*}{0.160} \\
\hline & $\mathrm{T} 1$ & $79.99 \pm 7.06$ & & $78.68 \pm 5.11$ & & $78.30 \pm 4.39$ & & \\
\hline & T1-T0 & $-0.65 \pm 1.66$ & & $0.40 \pm 2.05$ & & $0.37 \pm 1.44$ & & \\
\hline \multirow{3}{*}{$\begin{array}{l}\text { VRL-ls } \\
(\mathrm{mm})\end{array}$} & T0 & $82.65 \pm 7.95$ & \multirow{3}{*}{0.113} & $80.74 \pm 5.68$ & \multirow{3}{*}{0.649} & $79.55 \pm 5.25$ & \multirow{3}{*}{0.130} & \multirow{3}{*}{0.091} \\
\hline & $\mathrm{T} 1$ & $81.66 \pm 8.21$ & & $80.44 \pm 5.19$ & & $80.57 \pm 5.12$ & & \\
\hline & T1-T0 & $-0.99 \pm 2.32$ & & $-0.30 \pm 2.37$ & & $1.02 \pm 2.16$ & & \\
\hline \multirow{3}{*}{ E-UL (mm) } & T0 & $-3.26 \pm 1.94$ & \multirow{3}{*}{$0.027 *$} & $-3.36 \pm 2.35$ & \multirow{3}{*}{0.062} & $-3.93 \pm 2.44$ & & \\
\hline & $\mathrm{T} 1$ & $-4.04 \pm 2.45$ & & $-4.19 \pm 1.92$ & & $-3.65 \pm 2.36$ & 0.897 & 0.151 \\
\hline & T1-T0 & $-0.78 \pm 1.24$ & & $-0.83 \pm 1.62$ & & $0.28 \pm 1.61$ & & \\
\hline & T0 & $-1.28 \pm 2.19$ & & $-0.87 \pm 2.02$ & & $-2.20 \pm 1.84$ & & $<0.001 *$ \\
\hline E-LL (mm) & $\mathrm{T} 1$ & $-2.78 \pm 2.64$ & $0.003 *$ & $-2.41 \pm 2.12$ & $0.008 *$ & $-1.39 \pm 1.74$ & 0.052 & $(1-3=0.001)$ \\
\hline & T1-T0 & $-1.50 \pm 1.69$ & & $-1.54 \pm 1.70$ & & $0.81 \pm 1.60$ & & $(2-3=0.002)$ \\
\hline & T0 & $11.32 \pm 1.71$ & & $11.80 \pm 2.20$ & & $12.15 \pm 2.24$ & & $<0.001 *$ \\
\hline U1-ls (mm) & $\mathrm{T} 1$ & $13.01 \pm 1.77$ & $<0.001 *$ & $13.80 \pm 2.00$ & $0.001 *$ & $11.37 \pm 1.83$ & 0.063 & $(1-3<0.001)$ \\
\hline & T1-T0 & $1.69 \pm 1.22$ & & $2.00 \pm 1.82$ & & $-0.78 \pm 1.74$ & & $(2-3<0.001)$ \\
\hline & T0 & $12.27 \pm 2.30$ & & $13.31 \pm 1.39$ & & $12.68 \pm 2.37$ & & $0.003^{*}$ \\
\hline L1-li (mm) & $\mathrm{T} 1$ & $12.87 \pm 2.37$ & $0.032 *$ & $13.97 \pm 1.29$ & 0.075 & $11.97 \pm 1.35$ & $0.029 *$ & $(1-3=0.010)$ \\
\hline & T1-T0 & $0.60 \pm 1.00$ & & $0.66 \pm 1.27$ & & $-0.71 \pm 1.48$ & & $(2-3=0.009)$ \\
\hline & T0 & $21.52 \pm 2.29$ & & $22.24 \pm 3.61$ & & $19.75 \pm 1.99$ & & \\
\hline Upper lip & $\mathrm{T} 1$ & $21.83 \pm 2.79$ & 0.351 & $22.79 \pm 2.40$ & 0.210 & $20.38 \pm 1.89$ & 0.185 & 0.702 \\
\hline & T1-T0 & $0.31 \pm 1.21$ & & $0.56 \pm 1.65$ & & $0.62 \pm 1.75$ & & \\
\hline & T0 & $79.69 \pm 8.88$ & & $78.14 \pm 5.46$ & & $77.04 \pm 5.86$ & & $0.001 *$ \\
\hline VRL-li (mm) & $\mathrm{T} 1$ & $78.07 \pm 8.73$ & $0.010 *$ & $77.23 \pm 5.05$ & 0.268 & $78.55 \pm 5.55$ & $0.005^{*}$ & $(1-3=0.001)$ \\
\hline & T1-T0 & $-1.62 \pm 2.23$ & & $-0.91 \pm 2.96$ & & $1.51 \pm 1.71$ & & $(2-3=0.013)$ \\
\hline VRI_B' & T0 & $71.49 \pm 9.18$ & & $69.44 \pm 5.76$ & & $68.71 \pm 6.28$ & & $0.009 *$ \\
\hline VRL-B & $\mathrm{T} 1$ & $70.57 \pm 9.04$ & 0.196 & $68.02 \pm 5.33$ & 0.113 & $69.97 \pm 6.03$ & $0.011 *$ & $(1-3=0.044)$ \\
\hline & T1-T0 & $-0.92 \pm 2.36$ & & $-1.42 \pm 3.08$ & & $1.26 \pm 1.67$ & & $(2-3=0.013)$ \\
\hline VRL-pog' & T0 & $70.44 \pm 10.37$ & & $68.64 \pm 6.07$ & & $68.56 \pm 7.34$ & & $0.018 *$ \\
\hline$(\mathrm{mm})$ & $\mathrm{T} 1$ & $70.25 \pm 10.37$ & 0.569 & $68.50 \pm 5.85$ & 0.572 & $70.12 \pm 7.17$ & $0.010 *$ & $(1-3=0.047)$ \\
\hline & T1-T0 & $-0.19 \pm 1.43$ & & $-0.14 \pm 2.10$ & & $1.57 \pm 2.06$ & & $(2-3=0.039)$ \\
\hline & T0 & $127.35 \pm 3.44$ & & $129.56 \pm 2.87$ & & $126.52 \pm 4.10$ & & \\
\hline na-prn-pog ${ }^{\circ}$ & $\mathrm{T} 1$ & $127.42 \pm 4.19$ & 0.638 & $129.23 \pm 4.02$ & 0.556 & $126.97 \pm 3.90$ & 0.507 & 0.848 \\
\hline & T1-T0 & $0.07 \pm 2.14$ & & $-0.33 \pm 2.46$ & & $0.45 \pm 2.19$ & & \\
\hline & T0 & $157.78 \pm 5.14$ & & $159.54 \pm 5.70$ & & $156.18 \pm 4.82$ & & \\
\hline na-sn-pog ${ }^{\circ}$ & $\mathrm{T} 1$ & $158.55 \pm 5.55$ & 0.438 & $159.29 \pm 6.60$ & 1.000 & $157.14 \pm 3.95$ & 0.163 & 0.548 \\
\hline & T1-T0 & $0.77 \pm 3.60$ & & $-0.26 \pm 3.11$ & & $0.96 \pm 3.01$ & & \\
\hline & $\mathrm{T0}$ & $103.87 \pm 13.39$ & & $105.56 \pm 11.76$ & & $105.81 \pm 9.02$ & & \\
\hline nasolabial & $\mathrm{T} 1$ & $104.85 \pm 12.44$ & 0.723 & $106.83 \pm 16.19$ & 0.737 & $102.68 \pm 6.31$ & $0.039 *$ & 0.246 \\
\hline & T1-T0 & $0.98 \pm 7.27$ & & $1.27 \pm 8.09$ & & $-3.13 \pm 6.66$ & & \\
\hline
\end{tabular}

Wilcoxon signed rank test was used for intragroup comparisons, Kruskal-Wallis test was used for intergroup comparisons, and Bonferroni Dunn test for post-hoc analysis. (1-2): $\mathrm{p}$ value for difference between group 1 and 2, (1-3): $\mathrm{p}$ value for difference between group 1 and 3, (2-3): $\mathrm{p}$ value for difference between group 2 and 3 .

$\mathrm{p}<0.05$ is statistically significant.

In relation to the E-plane, the lower lip was retracted by $1.50 \mathrm{~mm}$ in Group 1 and $1.54 \mathrm{~mm}$ in Group 2, but was protracted by $0.81 \mathrm{~mm}$ in Group 3. These changes in Groups 1 and 2 showed a statistically significant difference from Group 3 $(\mathrm{p}<0.001)$. According to the vertical reference line (VRL-li), the lower lip was retracted in both extraction groups, and showed a statistically significant change compared to the non-extraction group (an increase of $1.51 \mathrm{~mm})(\mathrm{p}=0.001)$. The mean change values for the upper and lower lip thicknesses were $1.69 \mathrm{~mm}$ and $0.60 \mathrm{~mm}$ for Group 1 , and $2 \mathrm{~mm}$ and $0.66 \mathrm{~mm}$ for Group 2, respectively, both of which were greater than in Group 3 (decrease of $0.78 \mathrm{~mm}$ for upper lip thickness and $0.71 \mathrm{~mm}$ for lower lip thickness) $(\mathrm{p}<0.05)$. The mean soft tissue change values for VRL-B' and VRL-pog' showed a statistically significant increase in Group 3 compared to Groups 1 and $2(\mathrm{p}<0.05)$. Groups 1 and 2 did not 
show a significant difference in any dentoskeletal or soft tissue measurements between each other $(\mathrm{p}>0.05)$.

\section{DISCUSSION}

Orthodontists frequently encounter moderate anterior crowding in Class I malocclusions. ${ }^{24}$ In cases with 4-9 $\mathrm{mm}$ arch size/tooth size discrepancies, non-extraction and extraction treatments are possible, and the treatment plan depends on the hard and soft tissue characteristics of the patient. The extraction option is particularly used to relieve moderate to severe crowding and/or to lessen dental or dentoalveolar protrusion, while non-extraction treatment is usually preferred for minor skeletal and moderate dental crowding. ${ }^{25}$ The conflict surrounding the decision whether to extract or not is mostly related to the stability of treatment and its effects on the soft tissue profile. It is thought that the facial profile might be improved by decreasing facial convexity. ${ }^{26}$ This fact can be considered as the reason for preferring tooth extraction in borderline cases. Most studies, 2,412,20,27-29 have compared extraction treatment vs. non-extraction in regard to profile changes. However, there is no consensus as to whether soft tissue profile changes are different from orthodontic treatment with the first premolar extraction from those treated with second premolar extractions. Hence, this retrospective study was conducted to compare both extraction modalities to each other and as well as to a non-extraction control group.

For a meaningful comparison of the effects of extraction vs. non-extraction treatments on the facial profile, a high degree of homogeneity is necessary, especially regarding the growth parameter. The groups in the present study exhibited similar age, sex, and CVMS distribution, and this result can be considered as advantageous to control the confounding factors due to growth status. Also, the groups exhibited almost similar pre-treatment soft tissue characteristics. Therefore, the effect of known confounding factors related to the soft tissue profile was reduced, and any differences among the three groups at the end of the treatment might be mostly attributed to the treatment modality.
However, the initial vertical skeletal parameters differed, especially between Group 2 and 3, and this difference can be related to the factor that the clinicians might have a tendency to extract second premolars instead of first premolars, for reducing the vertical height.

According to the results of the present study, the null hypothesis was accepted, and there were no statistically significant differences in soft tissue, dental and vertical facial height changes when comparing the extraction of four first premolars to four second premolars in Class I patients. Therefore, this study does not support that there is a greater retraction of the upper and lower lips when first premolar teeth are extracted compared to when second premolar teeth are removed, in contrary to the Nance's argument. ${ }^{30}$ Omar et al. ${ }^{22}$ compared first vs. second premolar extraction in soft tissue profile changes. Similar to the results of our study, Omar et al. ${ }^{22}$ did not observe significant differences between different extraction patterns, such as the nasolabial angle and upper and lower lip position changes. On the other hand, different from the results of the present study, Omar et al. ${ }^{22}$ found that the amount of retraction of upper and lower incisors achieved in second premolar extraction cases was less than half the amount of retraction achieved in first premolar extraction cases. In the present study, both extraction patterns showed similar significant retraction of the upper and lower incisor teeth. This difference may arise from different anchorage techniques since they used a molar anchorage appliance more frequently in the first premolar extraction group. On the other hand, the same moderate anchorage mechanics were used in both extraction groups in the present study.

The results of the present study indicated that both extraction groups showed statistically significant differences related to dental and soft tissue parameters when compared to the nonextraction control group. Lip retrusion is expected to be further increased in the case of extractions with active incisor retraction. ${ }^{31}$ In the present study, the lower lip showed statistically significant retraction instead of upper lip according to E-plane and VRL in both extraction 
groups. The significant retraction of lower lips in both the first and second premolar extraction groups, when compared to the non-extraction group, can be attributed to the posterior dentoalveolar movement of both upper and lower anterior segments. Kouli et al. ${ }^{29}$ evaluated facial profiles and hard tissue changes in matched extraction and non-extraction Class I patients with the use of discriminant analysis validation. Kouli et al. ${ }^{29}$ concluded that both lips, but especially the lower, were more retruded relative to the nose and chin, compared to the non-extraction group, similar to our results.

Similarly, the distance from the lower lip to the aesthetic line significantly increased more in the extraction group due to incisor retraction compared to the non-extraction group in the study by Kirschneck et al. ${ }^{4}$ Yashwant et al. ${ }^{28}$ compared soft tissue changes in Class I borderline patients treated with either extraction or non-extraction modalities. According to their results, upper and lower lips were more retracted, and the thickness of the upper lip increased more in borderline extraction cases. In the present study, both upper and lower lip thicknesses significantly increased in both extraction groups compared to the nonextraction group, and might be related to the loss of tension in the upper and lower lips following the retraction of anterior teeth. According to the results of the present study, the nasolabial angle did not show a significant change in extraction groups, similar to the study by Kirschneck et al. ${ }^{4}$, contrary to the results of other studies ${ }^{20,28,32}$ that showed a significant increase in this angle. The differences in soft tissue changes, due to extraction or non-extraction treatment between different studies, may depend on the characteristics of the patients studied, sample size, the prescription used, anchorage considerations, and treatment mechanics.

In the present study, we also compared the effect of four premolar extractions, for the skeletal vertical dimension. The main idea behind this supposition is that tooth extractions reduce the vertical dimension based on the wedge-effect concept, by the anterior rotation of the mandible. ${ }^{33}$ Also, in the non-extraction treatment protocol, it could be expected that vertical facial height might increase because of the buccal crown tipping in the posterior area during crowding relief. However, the results of the present study did not show a significant change of vertical height in either the extraction or non-extraction groups. A recent systematic review ${ }^{34}$ assessed the effects of orthodontic treatment with four premolar extractions on the skeletal vertical dimension compared to non-extraction treatment. This review concluded that an extraction treatment protocol aiming to reduce vertical dimension does not seem to be an evidence-based clinical approach, as several studies ${ }^{35-37}$ indicated no significant differences between extraction and non-extraction treatments. In the study by Beit $e t$ $a l .{ }^{38}$, the extraction of four first premolars showed a slight decrease in the vertical skeletal measurements, whereas non-extraction treatment showed a slight increase. However, Beit et al. ${ }^{38}$ commented that because of the small-scale intergroup differences, it is open to discuss whether these results are clinically significant. Similar to our study design and results, Kim et $a l .{ }^{21}$ suggested that there was no decrease in facial vertical dimension regardless of the maxillary and mandibular first or second premolar extractions.

From the findings of the present study, the clinical relevance for orthodontists is that during orthodontic treatment, including an extraction protocol, extraction of either the four first or four second premolar teeth does not show different impacts on the facial profile and vertical parameters when using the same moderate anchorage mechanics. Therefore, the clinician should consider other possible factors, such as tooth prognosis, morphology, or size, while deciding whether to extract the first or second premolar teeth.

Some study design bias factors need to be considered when interpreting the results of this study. The possible growth changes in the nose, lips, and chin were not measured. Because of the nature of the study, the premolar extraction pattern was not determined randomly. It would be better to perform well-conducted randomized clinical trials with large sample sizes to increase 
the confidence of evidence regarding the effect of different extraction patterns on soft tissue profiles.

\section{CONCLUSIONS}

- The change in soft tissue profile and dentoskeletal parameters following extraction treatment was similar, regardless of whether the first premolar or second premolar teeth were extracted.

- Maxillary and mandibular incisor inclinations significantly decreased in extraction groups, compared to the non-extraction group.

- In relation to the E-plane, the lower lip was retracted by $1.50 \mathrm{~mm}$ and $1.54 \mathrm{~mm}$ for Groups 1 and 2, and protracted by $0.81 \mathrm{~mm}$ for Group 3, respectively.

- The lower lip was retracted by $1.62 \mathrm{~mm}$ and $0.91 \mathrm{~mm}$, according to the VRL in Groups 1 and 2 , respectively, and protracted by $1.51 \mathrm{~mm}$ in Group 3.

\section{ACKNOWLEDGEMENTS}

None

\section{CONFLICT OF INTEREST STATEMENT}

The authors declare that they have no competing interests.

Farklı Premolar Çekimli Ortodontik Tedavi

\section{Yöntemlerinin Yumuşak Doku Profili Bakımından}

\section{Karşılaş̧ırılması}

\section{$\ddot{O} Z$}

Amaç: Farkl premolar çekimli ve çekimsiz tedavi yöntemleri arasında yumuşak doku profili ve dentoiskeletsel parametrelerdeki değişikliklerin karşılaştırılmasıdır. Gereç ve Yöntemler: İskelet Sınıf I maloklüzyona sahip 50 hasta üç gruba ayrıldl. Grup 1, maksiller ve mandibular birinci premolar çekimiyle

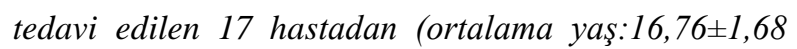
yll); Grup 2, maksiller ve mandibular ikinci premolar çekimiyle tedavi edilen 16 hastadan (ortalama

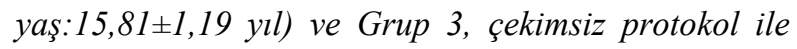
tedavi edilen 17 hastadan (ortalama yaş:16,29 $\pm 1,15$ yıl) oluşuyordu. Tedavi öncesi (T0) ve tedavi sonrası (T1) lateral sefalometrik radyograflar değerlendirildi. Dentoiskeletsel parametreler için 13, yumuşak doku parametreleri için 15 ölçüm yapıldı. Tedaviye bağlı yumuşak doku ve dentoiskeletsel değişiklikleri belirlemek ve gruplar arasındaki farklılıkları karşılaştırmak için sırasıyla Wilcoxon İşaretli Sıra ve
Kruskal-Wallis testleri uyguland. Bulgular: Mx1-SN, Mx1-FH, Mx1-NA, IMPA ve Md1-NB değerleri Grup 1 ve 2'de Grup 3'e göre anlaml olarak azald ( $p$ <0,001). Vertikal referans çizgisine (VRL-li) ve Edüzlemine (E-LL) göre, alt dudak Grup 1 ve 2'de çekimli olmayan gruba klyasla istatistiksel olarak anlamll bir değişiklik (retraksiyon) gösterdi $(p<0,05)$. Grup 1 ve 2'deki üst ve alt dudak kalınlıkları için ortalama değişim değeri Grup 3'ten daha fazlayd $(p<0,05)$. Dentoiskeletsel ve yumuşak doku ölçümleri bakımından Grup 1 ve 2 arasinda istatistiksel olarak anlamlı bir farklılık bulunmadl. Sonuçlar: Çekimli tedavi sonrası yumuşak doku profilindeki değişiklik, birinci veya ikinci premolar dişlerin çekilip çekilmediğine bakılmaksızın benzerdi. Ancak, çekimli tedavi yöntemleri, çekimsiz kontrol grubuna göre özellikle alt dudak bölgesinde önemli profil değişikliklerine neden oldu. Anahtar Kelimeler: Dikey boyut, diş çekimi, yumuşak doku.

\section{REFERENCES}

1. Bishara SE, Cummins DM, Jakobsen JR. The morphologic basis for the extraction decision in Class II, division 1 malocclusions: a comparative study. Am J Orthod Dentofacial Orthop 1995;107:129-135.

2. Erdinc AE, Nanda RS, Dandajena TC. Profile changes of patients treated with and without premolar extractions. Am J Orthod Dentofacial Orthop 2007;132:324-331.

3. Germec-Cakan D, Taner TU, Akan S. Arch-width and perimeter changes in patients with borderline Class I malocclusion treated with extractions or without extractions with air-rotor stripping. Am J Orthod Dentofacial Orthop 2010;137:734 e1-7; discussion-5.

4. Kirschneck C, Proff P, Reicheneder C, Lippold C. Short-term effects of systematic premolar extraction on lip profile, vertical dimension and cephalometric parameters in borderline patients for extraction therapy--a retrospective cohort study. Clin Oral Investig 2016;20:865-874.

5. Herzog C, Konstantonis D, Konstantoni N, Eliades T. Arch-width changes in extraction vs nonextraction treatments in matched Class I borderline malocclusions. Am J Orthod Dentofacial Orthop 2017;151:735-743.

6. Weyrich C, Lisson JA. The effect of premolar extractions on incisor position and soft tissue profile in 
patients with Class II, Division 1 malocclusion. J Orofac Orthop 2009;70:128-138.

7. Germec D, Taner TU. Effects of extraction and nonextraction therapy with air-rotor stripping on facial esthetics in postadolescent borderline patients. Am J Orthod Dentofacial Orthop 2008;133:539-549.

8. Lim HJ, Ko KT, Hwang HS. Esthetic impact of premolar extraction and nonextraction treatments on Korean borderline patients. Am J Orthod Dentofacial Orthop 2008;133:524-531.

9. Basciftci FA, Uysal T, Buyukerkmen A, Demir A. The influence of extraction treatment on Holdaway soft-tissue measurements. Angle Orthod 2004;74:167173.

10. Bowman SJ, Johnston LE, Jr. The esthetic impact of extraction and nonextraction treatments on Caucasian patients. Angle Orthod 2000;70:3-10.

11. Drobocky OB, Smith RJ. Changes in facial profile during orthodontic treatment with extraction of four first premolars. Am J Orthod Dentofacial Orthop 1989;95:220-230.

12. Young TM, Smith RJ. Effects of orthodontics on the facial profile: a comparison of changes during nonextraction and four premolar extraction treatment. Am J Orthod Dentofacial Orthop 1993;103:452-458.

13. Pearson LE. Vertical control in treatment of patients having backward-rotational growth tendencies. Angle Orthod 1978;48:132-140.

14. Schudy FF. The Rotation of the Mandible Resulting from Growth: Its Implications in Orthodontic Treatment. Angle Orthod 1965;35:36-50.

15. Schudy FF. The control of vertical overbite in clinical orthodontics. Angle Orthod 1968;38:19-39.

16. Tulley WJ. The role of extractions in orthodontic treatment. Br Dent J 1959;107:199-205.

17. Bishara SE, Jakobsen JR. Profile changes in patients treated with and without extractions: assessments by lay people. Am J Orthod Dentofacial Orthop 1997;112:639-644.

18. Stephens CK, Boley JC, Behrents RG, Alexander RG, Buschang PH. Long-term profile changes in extraction and nonextraction patients. Am J Orthod Dentofacial Orthop 2005;128:450-457.
19. Hayashida H, Ioi H, Nakata S, Takahashi I, Counts AL. Effects of retraction of anterior teeth and initial soft tissue variables on lip changes in Japanese adults. Eur J Orthod 2011;33:419-426.

20. Konstantonis D. The impact of extraction vs nonextraction treatment on soft tissue changes in Class I borderline malocclusions. Angle Orthod 2012;82:209-217.

21. Kim TK, Kim JT, Mah J, Yang WS, Baek SH. First or second premolar extraction effects on facial vertical dimension. Angle Orthod 2005;75:177-182.

22. Omar Z, Short L, Banting DW, Saltaji H. Profile changes following extraction orthodontic treatment: A comparison of first versus second premolar extraction. Int Orthod 2018;16:91-104.

23. Illing HM, Morris DO, Lee RT. A prospective evaluation of Bass, Bionator and Twin Block appliances. Part I--The hard tissues. Eur J Orthod 1998;20:501-516.

24. Oden F, Bekar E, Bıçakcı AA. Evaluation of malocclusion and crowding in under orthodontic treatment. Cumhuriyet Dent J 2015;18:257-264.

25. Konstantonis D, Vasileiou D, Papageorgiou SN, Eliades T. Soft tissue changes following extraction vs. nonextraction orthodontic fixed appliance treatment: a systematic review and meta-analysis. Eur J Oral Sci 2018;126:167-179.

26. Trakyalı G, Dayığlu N. Compliance and effectiveness in Cervical Headgear. Cumhuriyet Dent $\mathbf{J}$ 2019; 22:147-155.

27. Kocadereli I. Changes in soft tissue profile after orthodontic treatment with and without extractions. Am J Orthod Dentofacial Orthop 2002;122:67-72.

28. Yashwant VA RK, Arumugam E. Comparative evaluation of soft tissue changes in Class I borderline patients treated with extraction and nonextraction modalities. Dental Press J Orthod 2016;21:50-59.

29. Kouli A, Papagiannis A, Konstantoni N, Halazonetis DJ, Konstantonis D. A geometric morphometric evaluation of hard and soft tissue profile changes in borderline extraction versus non-extraction patients. Eur J Orthod 2018;41:264-272.

30. Nance HN. The removal of second premolars in orthodontic treatment. Am J Orthod 1949;35:685-696. 
31. Hodges A, Rossouw PE, Campbell PM, Boley JC, Alexander RA, Buschang PH. Prediction of lip response to four first premolar extractions in white female adolescents and adults. Angle Orthod 2009;79:413-421.

32. Ramos AL, Sakima MT, Pinto Ados S, Bowman SJ. Upper lip changes correlated to maxillary incisor retraction--a metallic implant study. Angle Orthod 2005;75:499-505.

33. Fields HW, Proffit WR, Nixon WL, Phillips C, Stanek E. Facial pattern differences in long-faced children and adults. Am J Orthod 1984;85:217-223.

34. Kouvelis G, Dritsas K, Doulis I, Kloukos D, Gkantidis N. Effect of orthodontic treatment with 4 premolar extractions compared with nonextraction treatment on the vertical dimension of the face: A systematic review. Am J Orthod Dentofacial Orthop 2018;154:175-187.
35. Kocadereli I. The effect of first premolar extraction on vertical dimension. Am J Orthod Dentofacial Orthop 1999;116:41-45.

36. Kumari M, Fida M. Vertical facial and dental arch dimensional changes in extraction vs. non-extraction orthodontic treatment. J Coll Physicians Surg Pak 2010;20:17-21.

37. Basciftci FA, Usumez S. Effects of extraction and nonextraction treatment on class I and class II subjects. Angle Orthod 2003;73:36-42.

38. Beit $\mathrm{P}$, Konstantonis D, Papagiannis A, Eliades $\mathrm{T}$. Vertical skeletal changes after extraction and nonextraction treatment in matched class I patients identified by a discriminant analysis: cephalometric appraisal and Procrustes superimposition. Prog Orthod 2017;18:44. 